\title{
Luxación volar irreductible aislada de la extremidad distal del cúbito
}

\author{
J. A. Cara del $\operatorname{Rosal}^{(1)}$, L. Martos ${ }^{(2)}$, E. Navarro ${ }^{(3)}$ \\ ${ }^{(1)}$ Servicio Cirugía Ortopédica y Traumatología Y ${ }^{(2)}$ Rehabilitación, ${ }^{(3)}$ Servicio de Radiología del \\ Hospital Costa del Sol.
}

Correspondencia:

Dr. José Antonio Cara del Rosal

Servicio de Cirugía Ortopédica y Traumatología

Hospital Costa del Sol

C.N. 340, Km. 187

29600 Marbella (Málaga)

E-mail: jac.cara@teleline.es

Las luxaciones de la articulación radiocubital distal se asocian con fracturas del radio. La luxación aislada de la articulación radiocubital distal es rara, suponiendo menos del $0,2 \%$ de todas las luxaciones, y se describe terminológicamente por la posición que adopta el cúbito respecto al radio. Radiológicamente pueden pasar desapercibidas por lo que a veces es necesario el uso de la Tomografía Axial Computerizada. Presentamos el caso de un varón de 37 años que sufrió una luxación volar irreductible de la extremidad distal del cúbito. Fue intervenido de urgencia mediante reducción abierta y estabilización con aguja de Kirschner, observando una interposición de los ligamentos radiocubitales dorsales que era lo que impedía la reducción cerrada. A las 6 semanas se retiró la aguja, comenzando con la rehabilitación. Se realizó control radiológico y TAC en pronación y supinación observando una buena congruencia. Clínicamente está asintomático.
Luxations of the distal radioulnar joint are associated with fractures of the radial. The isolated luxation of the distal radioulnar joint is rare, making up less than $0.2 \%$ of all luxations, and is described terminologically by the position adopted by the ulnar with respect to the radial. They can escape the attention of radiology and so it is sometimes necessary to use Computerised Axial Tomography. We present the case of a 37-year-old male who suffered a volar luxation of the distal extremity of the ulnar. He was treated urgently through open reduction and stabilisation with a Kirschner needle, and an interposition of the dorsal radioulnar ligaments was observed, which is what prevented closed reduction. After 6 weeks the needle was removed and rehabilitation began. Radiological and CAT monitoring in pronation and supination was carried out, and good congruence was observed. He now presents no clinical symptoms.

\section{INTRODUCCIÓN}

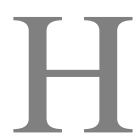
abitualmente las luxaciones de la articulación radiocubital distal (ARCD) se asocian con fracturas de la extremidad distal del radio o con lesiones más complejas (fractura-luxación de Galeazzi) (Bruckner, Ale- xander y Lichtman, 1995, y Geissler, Fernández y Lamey, 1996). La luxación aislada de la ARCD es rara, suponiendo menos del $0,2 \%$ de todas las luxaciones (Fehring, T.K. y Milek, 1984) y se describe terminológicamente por la posición que adopta el cúbito respecto a dicha articulación. Radiológicamente pueden pasar 

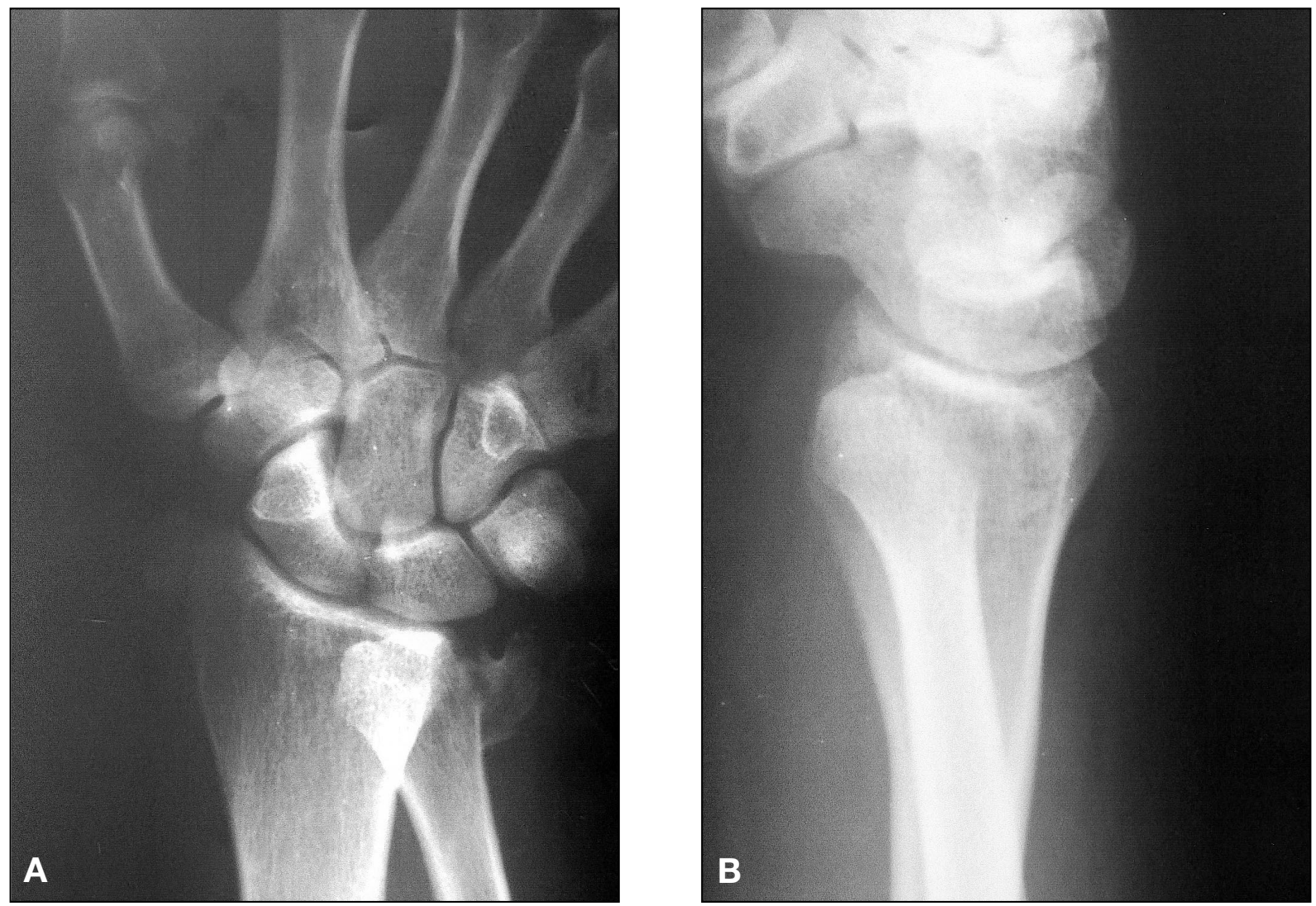

Figura 1. Imagen anteroposterior (A) y lateral (B) de la luxación. Obsérvese cómo en la imagen lateral tiene una apariencia normal y en la anteroposterior se observa la superposición del radio y del cúbito.

desapercibidas si no se realiza una radiografía lateral correcta (Mino, Plamer y Levinsohn, 1985).

Presentamos el caso de una luxación volar de la extremidad distal del cúbito tratada de forma aguda.

\section{CASO CLÍNICO}

Varón de 37 años que acude a urgencias tras caída casual sobre la muñeca derecha con el antebrazo supinado. Presentaba molestias en la zona, sin evidencia de deformidad alguna. A la exploración del balance articular, tenía una movilización de la muñeca en flexo-extensión completa, con bloqueo para la pronosupinación con la mano fija en supinación.

Se realizó estudio radiológico, observando una incongruencia entre la imagen anteroposterior y lateral (Figura 1) por lo que se aconsejó realizar TAC (Figura 2), observando una lu- xación volar del cúbito con fractura de la estiloides cubital.

Se llevó a quirófano y se hizo un intento de reducción cerrada que no fue posible, por lo que se realizó una reducción abierta de la luxación, estabilizándola con una aguja Kirschner en posición neutra. Durante el acto operatorio se observó cómo los ligamentos dorsales de la ar-

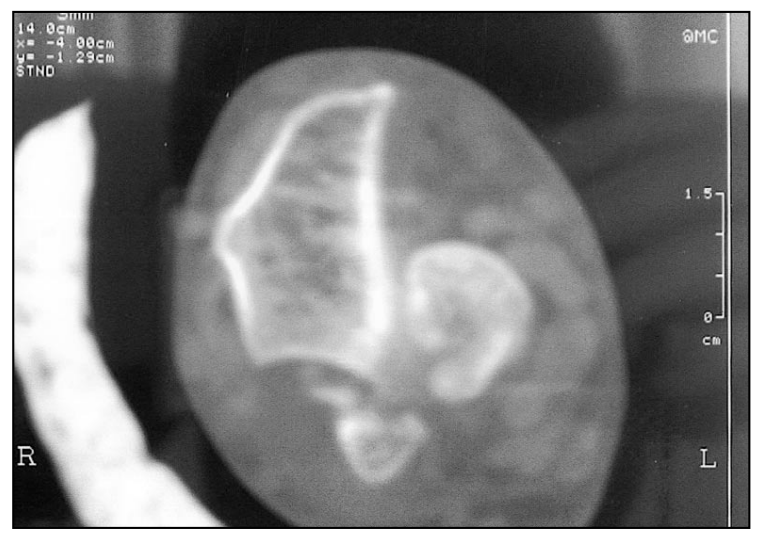

Figura 2. Imagen del TAC donde se observa la luxación volar y la fractura de la estiloides cubital. 

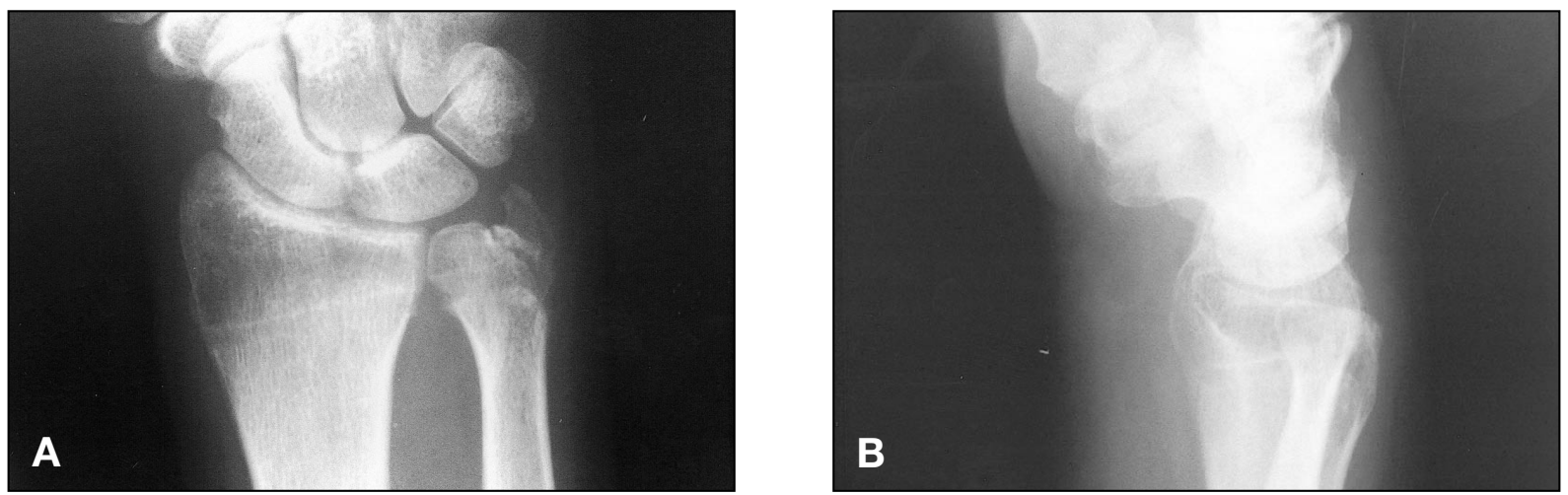

Figura 3. Imagen anteroposterior (A) y lateral (B) donde se observa la reducción y consolidación de la fractura.

ticulación radiocubital distal etaban interpuestos e impedían la reducción.

A las 6 semanas se retiró la aguja comenzando con la rehabilitación.

Se realizó control radiológico (Figura 3) y TAC en pronación y supinación (Figura 4), observando una buena congruencia de la articulación. Clínicamente el paciente está asintomático con movilidad completa.

\section{DISCUSIÓN}

La articulación radiocubital distal y su patología es compleja (Amillo, 1993; Bruckner, Alexander y Lichtman, 1995; García-Elías, 1999, y Geissler, Fernández y Lamey, 1996). La mayoría de las luxaciones radiocubitales distales se asocian a fracturas de los huesos del antebrazo (Bruckner, Alexander y Lichtman, 1995, y Geissler, Fernández y Lamey, 1996). Las luxaciones aisladas son menos frecuentes y pue- den pasar desapercibidas (García Olea et al., 1997). Una posible explicación para esto es que en pacientes que tienen una luxación volar, la prominencia que suele producir la cabeza del cúbito desaparece, por lo que el diagnóstico es menos obvio que cuando la prominencia de la cabeza cubital aumenta en las luxaciones o subluxaciones dorsales. A veces es difícil realizar el diagnóstico en un examen radiológico rutinario. Una proyección lateral correcta es difícil de realizar, ya que el paciente tiene el bloqueo para la pronosupinación. La imagen dada por la TAC es la más exacta para llegar al diagnóstico (Mino, Palmer y Levisnsohn, 1985).

La luxación volar suele ocurrir tras un traumatismo en el que se fuerza la supinación (Brucker, Alexander y Lichtman, 1995). En el traumatismo se suelen lesionar los ligamentos dorsales radiocubitales y de la cápsula palmar de dicha articulación. La luxación suele reducirse colocando el antebrazo en pronación. En nuestro caso no fue posible la reducción abier-
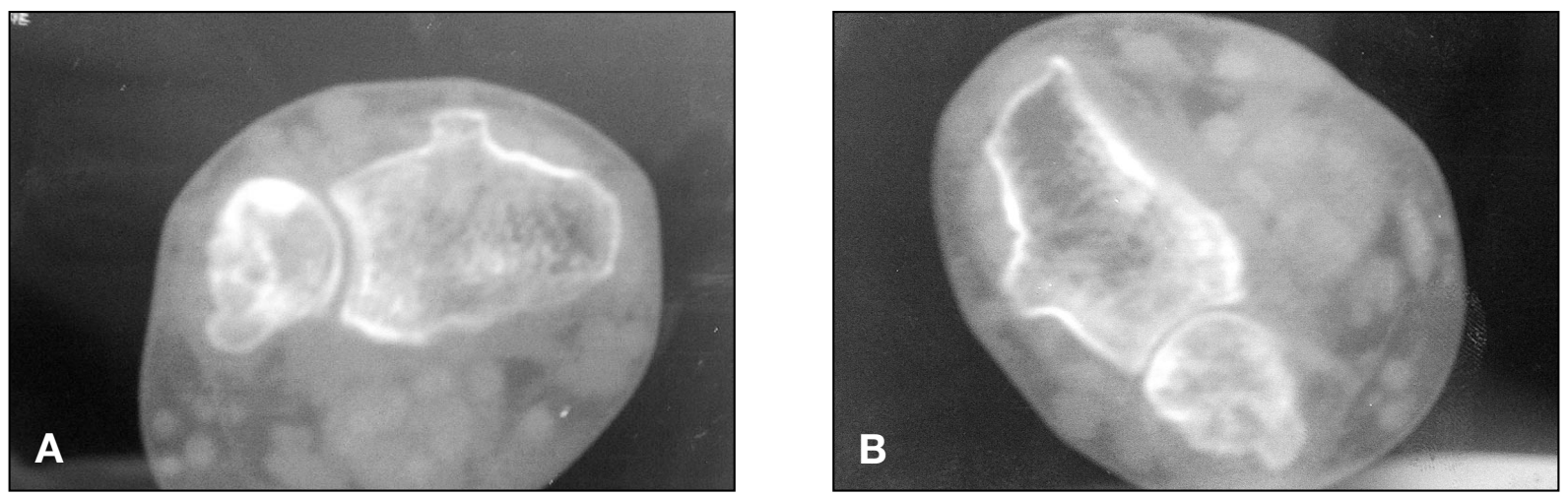

Figura 4. Imagen de TAC en pronación (A) y supinación (B). Obsérvese la buena congruencia articular. 
ta, ya que los ligamentos dorsales de la articulación radiocubital distal estaban incarcerados e impedían la reducción de la luxación.

En la mayoría de los trabajos publicados los pacientes fueron intervenidos varias semanas después del traumatismo, ya que éste pasó desapercibido (García Olea et al., 1997, y Schiller M. G., afEkenstam y Kirch, 1991). En nuestro caso, fue la imagen radiológica anteroposterior la que nos llamó la atención, ya que la lateral era considerada casi como normal; posteriormente, la TAC confirmó el diagnóstico.

Tras la inmovilización, que se aconseja que sea de 6 semanas, el programa de rehabilitación ha de ser intensivo, ya que la rigidez para la prono-supinación suele ser importante (Graham, McCoy y Mollan, 1985). En nuestro caso se realizó un programa que consiguió unos resultados buenos con una pronación de $90^{\circ}$ y supinación de $70^{\circ}$ (Skirven, 1997).
En resumen, la luxación dorsal aislada de la articulación radiocubital distal normalmente produce una lesión del complejo ligamentoso dorsal que puede bloquear la reducción. La muñeca puede aparentar estar normal pero en supinación. La pronación está bloqueada y la flexoextensión son normales y sin dolor. Si no se realiza una radiografía lateral correcta, la lesión puede pasar desapercibida. La TAC confirma el diagnóstico.

La reducción cerrada suele ser habitual, bajo anestesia general, mediante la maniobra de pronación de la muñeca, estabilización con una aguja de Kirschner e inmovilización durante 6 semanas hasta que cicatricen los tejidos blandos, aunque en ciertos casos puede ser necesaria la reducción abierta por incarceración de los ligamentos radiocubitales dorsales (Schiller, M. G., efEkenstam y Kirsch, 1991).

\section{BIBLIOGRAFÍA}

Geissler, W. B.; Fernández, D. L.; Lamey, D. M.: Distal radioulnar joint injuries associated with fractures of the distal radius. Clin. Orthop., 1996; 327: 59-70.

Brucker, J. D.; Alexander, A. H.; Lichtman, D. M.: Acute dislocations of the distal radio-ulnar joint. J. Bone Joint Surg., 1995; 77A: 958-968.

Fehring, T. K.; Milek, M. A.: Isolated volar dislocation of the radiocarpal joint. A case report. J. Bone Joint Surg., 1984; 66A: 464-6.

Mino, D. E.; Palmer, A. K.; Levinsohn, E. M.: Radiography and computerized tomography in the diagnosis of incongruity of the distal radio-ulnar joint. A prospective study. J. Bone Joint Surg., 1985; 67A: 247-252.

García Olea, A.; Bartolomé del Valle, E.; Torres Coscoyuela, M., et al.: Luxación volar radiocubital distal inveterada. Tratamiento mediante técnica de Sauvé-Kapanji. Rev. Ortop. Traumatol., 1997; 41: 344-349.

Schiller, M. G.; afEkenstam, F.; Kirsch, P. T.: Volar dislocation of the distal radio-ulnar joint. A case Report. J. Bone Joint Surg., 1991; 73!: 617-9.
Amillo Garayoa, S.: Patología radiocubital distal. Rev. Ortop. Traumatol., 1993; 37 (suppl 1): 35-41.

García Elías, M: Radio-ulnar instability. Current Orthop., 1999; 13: 283-289.

Graham, H. K.; McKoy, G. F.; Mollan, R. A. B.: A new injury of the distal radio-ulnar joint. J. Bone Joint Surg., 1985; 67B: 302-304.

Skirven, T.: Rehabilitation following surgery for the distal radioulnar joint. Techniques in Hand and Upper Extremity Surgery, 1997; 1: 219-225. 